\title{
Teste de Especificidade Hospedeira de Phaedon confinis (Coleoptera, Chrysomelidae), um Potencial Agente de Biocontrole de Senecio brasiliensis (Asteraceae)
}

\author{
Julianne Milléo ${ }^{1}$, Jonathan Pena Castro ${ }^{1}$ \& José Henrique Pedrosa-Macedo²
}

1. Universidade Estadual de Ponta Grossa, e-mail: jumilleo@gmail.com (Autor para correspondência ${ }^{\bowtie}$ ), jonathan.penacastro@gmail.com. 2. Universidade Federal do Paraná, e-mail: johpema@netpar.com.br. Fomento: Conselho Nacional de Desenvolvimento Científico e Tecnológico (CNPQ); Instituto Nacional de Ciência e Tecnologia (INCT)

EntomoBrasilis 4 (2): 61-66 (2011)

Resumo. Senecio brasiliensis (Spreng.) Less quando ingerido por bovinos e eqüinos causa uma séria intoxicação, a seneciose. Devido a significativa perda econômica para a pecuária é interessante o controle com uso de insetos. Através de coletas sistêmicas e contínuas na planta observou-se que Phaedon confinis Klug, causa sérios danos à planta apresentando grande potencial como biocontrolador. O objetivo foi ampliar a 52 plantas os testes de especificidade hospedeira com larvas de $1^{\mathrm{o}}$ instar e adultos do crisomelídeo. Os insetos foram submetidos aos testes "sem chance de escolha" e de "múltipla escolha". Os seguintes resultados foram obtidos: "SEM CHANCE DE ESCOLHA" L1 - 52 plantas testadas: dano nulo 90,39\%; exploratório 5,77\%; fraco 1,92\%; e normal apenas no $S$. brasiliensis $1,92 \%$, onde $31,67 \%$ das larvas atingiram a fase adulta. "SEM CHANCE DE ESCOLHA" ADULTOS - 46 plantas testadas: em 82,60\% o dano foi nulo; 13,04\% exploratório; 2,17\% fraco; 2,17\% normal S. brasiliensis. O crisomelídeo ovipositou durante os dias de observação somente nas folhas de $S$. brasiliensis, 615 ovos com viabilidade de 73,01\%. No teste "MÚLTIPLA ESCOLHA" LARVAS - Nove plantas testadas: $66,67 \%$ dano nulo; $11,11 \%$ fraco; $11,11 \%$ exploratório; e $11,11 \%$ normal $S$. brasiliensis. Os resultados indicam que a alimentação normal, oviposição, sobrevivência e desenvolvimento de $P$. confinis estão restritos ao $S$. brasiliensis e contribuem para confirmar o seu potencial como agente de biocontrole.

Palavras-Chave: Controle biológico; maria-mole; pecuária; planta tóxica

\section{Host Specificity Test of Phaedon confinis (Coleoptera, Chrysomelidae), a Potential Biocontrol Agent of Senecio brasiliensis (Asteraceae)}

Abstract. Senecio brasiliensis (Spreng.) Less when ingested by cattle and horses, the plant causes seneciosis, a serious poisoning. Due to the great financial losses to cattle ranchers, controlling the plant using insects has become attractive. Systematic survey efforts have revealed that Phaedon confinis Klug causes serious damage to the plant, and may be a great biocontrol agent. The object was to extend the tests of host specificity to 52 plants using 1st larval instar and adult chrysomelid bettles. The insects were submitted to "no-choice" and "multiple-choice" tests. The following results were obtained: "NO-CHOICE" L1 - 52 plants tested: null 90.39\%; negligible damage 5.77\%; light 1.92\%; and normal in only S. brasiliensis 1.92\%, where $31.67 \%$ of larvae obtained an adult phase. "NO-CHOICE" ADULTS - 46 plants. Null damage was recorded in $82.60 \%$; $13.04 \%$ showed negligible damage; $2.17 \%$ light; $2.17 \%$ normal in S. brasiliensis. The chysomelids oviposited during observation days only on S. brasiliensis leaves. 615 eggs were oviposited with 73.01\% viability. "MULTIPLE CHOICE" LARVAE - nine plants tested. $66.67 \%$ null; $11.11 \%$ weak; $11.11 \%$ negligible damage; and $11.11 \%$ normal in $S$. brasiliensis. The results indicate that the normal diet, oviposition, survival and development of $P$. confinis is restricted to $S$. brasiliensis and corroborates its potential as a biocontrol agent.

Keywords: Biological control; cattle-raising; maria-mole; toxic plant

A espécie mais freqüente de Senecio no centro-sul do Brasil, Uruguai e Argentina é Senecio brasiliensis (Spreng.) Less, considerada planta invasora de culturas e pastagens. Por conter alcalóides pirrolizidínicos, esta planta quando ingerida causa uma lesão hepática progressiva, conhecida como seneciose, que atinge principalmente bovinos e eqüinos (RIET-Correa et al. 1993). Rissi et al. (2007) ao revisarem 2912 casos referentes a necropsias de bovinos, entre 1990 e 2005 no Rio Grande do Sul, concluíram que em 15,83\% a causa da morte foi devido à ingestão de plantas tóxicas. Dentre os casos diagnosticados de envenenamento por plantas, $56,14 \%$ foram atribuídos a espécies de Senecio.

Devido à letalidade causada pela ingestão de $S$. brasiliensis, é necessário o controle desta planta em áreas de pastagem. Estudos multidisciplinares vêm reunindo esforços de profissionais de diversas áreas visando o desenvolvimento de estratégias eficazes de controle e profilaxia da intoxicação por
Senecio spp. na região sul do país (IlHa et al. 2001).

O controle biológico com uso de insetos é uma alternativa que pode ser associada a outras técnicas de combate ao Senecio spp., e apesar de atuar mais lentamente do que o agrotóxico é de efeito prolongado e sem ser prejudicial ao gado e ao meio ambiente. Embora pouco conhecido no Brasil, o uso de insetos para o controle de plantas indesejáveis é amplamente utilizado em países como Estados Unidos (incluindo Havaí), Austrália, África do Sul, Canadá e outros (MCFADYen 1998; Lenteren 2008). Entre os casos de sucesso, destaca-se o controle biológico do Senecio jacobea L., no estado do Oregon (Estados Unidos). Técnicas usuais de controle químico, mecânico e cultural surtiram efeitos apenas localizados e, em 1974, o departamento de agricultura iniciou um programa de controle biológico que em 1987 reduziu quase 90\% da planta. Três inimigos naturais foram trazidos da Europa: Tyria jacobaeae Linnaeus (Lepidoptera: Arctiidae), Longitarsus jacobaeae (Waterhouse) (Coleoptera: 
Chrysomelidae) e Pegohylemia seneciella Meade (Diptera: Anthomyiidae). Destes insetos, o coleóptero foi o que melhor se estabeleceu sendo encontrado em praticamente todas as áreas (Mcevor et al. 1991; Coombs et al. 1997).

Para pesquisar potenciais agentes de biocontrole do $S$. brasiliensis, MENDES et al. (2005) realizaram inventariamento da entomofauna associada a esta planta, no município de Ponta Grossa, PR. Entre os insetos observados destacou-se a presença de um crisomelídeo identificado como Phaedon confinis Klug causando sérios danos à planta, alimentando-se tanto das folhas como do caule jovem, em sua fase larval e adulta. Segundo GAZI et al. (2008) é importante a utilização de insetos como agentes de controle biológico que apresentem tendência à monofagia e, ao fato das larvas e adultos utilizarem o mesmo recurso alimentar, associado à baixa taxa de dispersão.

Alguns autores enfatizam o potencial do grupo para atuar em controle biológico: "os crisomelídeos formam um grupo de insetos com hábito alimentar preferencialmente oligófago, sendo muito utilizados no controle de plantas indesejáveis pelo mundo todo" (Zhang et al. 2008); "o gênero Phaedon tem potencial como agente de biocontrole de ervas daninhas, pois a maioria das espécies alimenta-se de um número restrito de hospedeiros" (Cox 1996).

Um dos trabalhos mais relevantes em controle biológico de plantas indesejáveis com insetos no Brasil é o de Lucchin (1996). O autor determinou alguns aspectos biológicos e aplicou testes de especificidade a Phaedon pertinax Stal em Bidens pilosa L. concluindo que o inseto tem potencial no controle biológico da planta.

A partir do levantamento bibliográfico da espécie $P$. confinis foram encontrados registros do inseto associado a esta planta em Bertels (1953), Lima (1954), Silva et al. (1968) e HoFfMANN \& MosCARDi (1980; 1981). HoFfMANN \& MosCaRdi (1980; 1981) realizaram alguns testes de especificidade hospedeira com este inseto e obtiveram alguns resultados preliminares. Em 1980, observaram a amplitude e duração média dos diferentes estádios de desenvolvimento de $P$. confinis, assim como, realizaram teste de "múltipla escolha" com larvas e adultos, em 12 plantas pertencentes a família Asteraceae. Nos resultados parciais obtidos, o inseto mostrou-se específico somente a S. brasiliensis). HOFFMANN \& MOSCARDI (1981) aplicaram às larvas recém-eclodidas o teste "sem chance de escolha" em 24 plantas, reforçando os resultados anteriores.

Nopresentetrabalhoprocurou-seavaliara especificidade hospedeira de $P$. confinis, como biocontrolador de $S$. brasiliensis, em condições de laboratório. Para isto, foram testadas 52 plantas e os seguintes critérios observados: nível de dano às plantas, sobrevivência dos insetos e preferência de planta para oviposição. Os insetos foram submetidos a três tipos de testes de preferência alimentar: 1) Larva de $1^{0}$ instar - "sem chance de escolha"; 2) Larva de $1^{0}$ instar - "múltipla escolha" e 3) Adulto - "múltipla escolha".

\section{MATERIAL E MÉTODOS}

Adultos de $P$. confinis foram coletados manualmente sobre $S$. brasiliensis, na Fazenda Escola Capão da Onça, pertencente à Universidade Estadual de Ponta Grossa, localizada na cidade de Ponta Grossa, Paraná, Brasil $\left(25^{\circ} \mathrm{O} 6^{\prime} \mathrm{S} 50^{\circ} \mathrm{Oo}\right.$ 'W $)$. No laboratório, os insetos foram separados em sete casais por recipiente com capacidade de $250 \mathrm{~mL}$, contendo algodão umedecido com água e folhas de $S$. brasiliensis, sendo mantidos para obtenção de ovos. As etapas de coleta dos ovos e criação dos insetos até a fase adulta, seguiram a metodologia descrita em MiLLÉo et al. (2006).

Paraseleçãodasespéciesvegetaisseguiram-seoscritérios propostos por WAPSHERE (1974), usuais em testes para controle biológico de plantas, que consideram a filogenia, biogeografia e ecologia da espécie-alvo. Portanto, foram selecionadas plantas: a) filogeneticamente aproximadas, b) nas quais o agente foi registrado, c) localizadas próximas da planta indesejável, d) de interesse ecológico ou econômico na área onde o controle do S. brasiliensis é desejável. Participaram dos testes 47 espécies vegetais (Tabela 1), incluindo três tipos comerciais de Lactuca sativa L. (Asteraceae) e quatro variedades de Brassica oleracea L. (Brassicaceae), totalizando 52 plantas (Tabela 1). As espécies vegetais foram testadas de acordo com sua disponibilidade no campo.

Foram realizados em laboratório, na temperatura ambiente, dois tipos de testes de especificidade o teste "sem chance de escolha" e de "múltipla escolha". As larvas de $1^{\circ}$ instar de $P$. confinis foram submetidas ao teste "sem chance de escolha". Em seguida, foram ofertadas aos adultos, somente as plantas pelas quais as larvas demonstraram qualquer interesse alimentar em qualquer uma das repetições, pois os adultos são mais seletivos que as larvas (Lucchini 1996). Para as plantas em que houve algum indicativo de dano nos testes anteriores, aplicou-se o teste de "múltipla escolha" para larvas de $1^{\circ}$ instar. Aspectos específicos do ciclo de vida de $P$. confinis em $S$. brasiliensis não foram avaliados, pois se encontram descritos em Milléo et al. (2006).

Teste sem chance de escolha: Somente uma única espécie de planta foi oferecida para as larvas e adultos. Antes de serem utilizadas no experimento as folhas das 52 plantas testadas foram lavadas e deixadas para secar naturalmente, em seguida cortadas com tesoura, de forma a apresentar uma área quadrangular de $3 \mathrm{~cm}^{2}$.

a) Com larvas: os bioensaios foram desenvolvidos a uma temperatura média do ar de $22,24^{\circ} \mathrm{C} \pm 2,5^{\circ} \mathrm{C}$, utilizando seis repetições para cada planta, isto é, seis placas-de-Petri com dez larvas em cada uma, totalizando 60 larvas de $1^{\circ}$ ínstar (de o a 24 horas de vida). Asleituras foram realizadas a cada 48 horas durante todo período de viabilidade dos insetos. Foram considerados os seguintes parâmetros: nível de dano e sobrevivência (número e mudanças de fases/desenvolvimento).

b) Com adultos: os bioensaios foram desenvolvidos a uma temperatura média do ar de $21,42^{\circ} \mathrm{C} \pm 3^{\circ} \mathrm{C}$, utilizando cinco repetições para cada planta, isto é, cinco placas-de-Petri com quatro adultos em cada, totalizando 20 adultos (de o a 24 horas de emergência). Foram realizadas sete leituras a cada 48 horas, por um período de 20 dias, após este período os adultos sobreviventes foram descartados. Foram analisados os seguintes parâmetros: nível de dano, porcentagem de sobreviventes, oviposição e viabilidade dos ovos. Para compor este teste foram utilizadas 46 plantas, inclusive algumas que tiveram resultado nulo, mas que as larvas mostraram algum indicativo de dano em alguma das repetições.

Múltipla escolha com larvas: os bioensaios foram desenvolvidos a uma temperatura média do ar de $17,3^{\circ} \mathrm{C} \pm$ $0,16^{\circ} \mathrm{C}$ e umidade relativa do ar de $59,7 \% \pm 5,6 \%$ utilizando seis repetições, com dez larvas (o a 24 horas de vida) cada uma. As nove plantas que obtiveram algum indicativo de dano nos dois testes anteriores foram novamente testadas. Em cada placa foram alocadas, além do $S$. brasiliensis, duas outras plantas escolhidas aleatoriamente, todas com área foliar de $3 \mathrm{~cm}^{2}$, dispostas de maneira eqüidistantes. As larvas de $1^{\mathrm{O}}$ ínstar foram posicionadas no centro da placa. As leituras foram realizadas a cada 48 horas, totalizando sete leituras ao final dos 20 dias de amostragem. A cada leitura, as folhas foram trocadas por novas. Neste teste foi analisado somente o nível de dano às plantas.

Para análise dos danos causados nas plantas foi adotada a escala visual adaptada de Lucchins (1996) com as seguintes categorias: Nulo (-) nenhum dano / o\%; Exploratório (+) dano insignificante / até 10\%; Fraco (++) dano fraco / até $30 \%$, poucas larvas atingem o $2^{\circ}$ instar; Moderado $(+++)$ dano moderado / até 50\%; Normal (++++) alimentação normal / mais de 50\%. Os resultados foram comparados com os trabalhos de Lucchini (1996) que aplicou testes de especificidade à Phaedon pertinax Stal, com vistas ao controle de $B$. pilosa; e de Hoffmann \& Moscardi $(1980 ; 1981)$. Ao final do experimento, os resultados dos testes de 
especificidade hospedeira de $P$. confinis (larvas e adultos) foram submetidos ao $\chi^{2}$, adotando-se um nível de significância de 5\%.

\section{RESULTADOS}

Teste "sem chance de escolha" com larvas. Foi observado que em 90,38\% das plantas testadas, ao realizar a média entre as repetições para cada planta, o resultado foi classificado como nulo. Não houve mudanças de instar e o tempo de sobrevivência das larvas encontra-se na tabela 1. Em 5,77\% plantas (Emilia sonchifolia (L.) DC Allium cepa L. e Brassica oleracea L. var. acephala) houve alimentação exploratória, não houve mudanças de instar e as larvas morreram até o quarto dia de contato com as folhas. O dano classificado como fraco foi observado em 1,92\% das plantas, B. oleracea var. italica, e das 60 larvas iniciais, $3,33 \%$ atingiram o $2^{\mathrm{o}}$ ínstar, sendo que nenhuma sobreviveu ao $3^{0}$ instar. Neste experimento não foi obtido nenhum resultado que pudesse ser classificado como moderado. Em S. brasiliensis $(1,92 \%)$ o dano foi classificado como normal; e das 60 larvas iniciais, $63,33 \%$ completaram o $1^{0}$ ínstar, 50,00\% completaram o $2^{\circ}$ ínstar e $31,67 \%$ completaram o $3^{\circ}$ ínstar, sobrevivendo até a fase adulta.

Teste "sem chance de escolha" com adultos. Por apresentarem resultado nulo em todas as repetições do teste anterior, Manihot utilissima Pohl, Psidium guajava L., Citrus limom (L.) Burm. F., Citrus reticulata Blanco, Citrus sinensis (L.) Osbeck e Lycopersicon esculentum Mill não foram incluídas neste teste.

Em relação aos danos causados e mortalidade dos adultos, das 46 plantas testadas, em 82,60\% não ocorreu nenhum tipo de dano na área foliar. O tempo máximo de sobrevivência observado foi de três adultos $(0,39 \%)$ até o $20^{\circ}$ dia, porém não causaram nenhum dano a estas plantas, sendo descartados após o término do experimento. Quando os adultos eram colocados em contato com as plantas, eles se dispersavam, ficando nas bordas das placas-de-Petri, ou próximos ao algodão umedecido. Em $B$. oleracea var. capitata, B. oleracea var. italica, $A$. cepa, B. oleracea var. botrytis, Brassica pekinensis L. e B. pilosa representando $13,04 \%$ do total das plantas testadas, houve alimentação exploratória. O dano classificado como fraco foi observado em $E$. sonchifolia, representando $2,17 \%$ das 46 plantas. Não foi obtido nenhum resultado que fosse classificado como moderado. Nos testes com $S$. brasiliensis, representando 2,17\% das plantas, os danos nas folhas foram classificados como normais devido à voracidade na alimentação, causando deterioração de grande parte da área foliar oferecida aos insetos.

A taxa de sobrevivência de adultos até o final dos 20 dias de teste foi de: $75 \% \mathrm{~S}$. brasiliensis, $65 \% \mathrm{E}$. sonchifolia, $45 \% \mathrm{~B}$. oleracea var. capitata, 40\% B. oleracea var. italica, 35\% A. cepa, $10 \%$ B. pilosa, $5 \%$ B. pekinensis e o\% B. oleracea var. acephala e B. oleracea var. botrytis.

$P$. confinis somente ovipositou nas folhas de $S$. brasiliensis, obtendo uma média de 127,20 ovos por placa-dePetri, durante os 20 dias de observação. De um total de 615 ovos, $88,13 \%$ foram postos na face abaxial das folhas, $9,11 \%$ na face adaxial e 2,76\% postos diretamente nas placas-de-Petri. Havendo uma viabilidade de $73,01 \%$, ou seja, 449 ovos, deram origem a larvas de $1^{\circ}$ instar.

Teste de "múltipla escolha" com larvas. As nove plantas que obtiveram resultado diferente de nulo nos testes anteriores, participaram do teste de "múltipla escolha". Em 66,67\% não ocorreu nenhum tipo de dano. Foi observado que assim que as larvas de $1^{\circ}$ instar foram colocadas no centro da placa, dirigiramse imediatamente ao $S$. brasiliensis, ignorando as demais plantas presentes. Uma vez estabelecidas no $S$. brasiliensis, as larvas agregavam-se até o ínstar seguinte. Em B. oleracea var. itálica e $A$. cepa, as larvas continuaram a apresentar algum tendência alimentar sendo os danos classificados como fraco $(11,11 \%)$ e exploratório (11,11\%), respectivamente. Apenas em S. brasiliensis o dano foi classificado como normal (11,11\%).

Os resultados dos três tipos de testes de especificidade hospedeira indicaram que as categorias de dano ocasionado por $P$. confinis (larvas e adultos) foram dependentes da espécie vegetal oferecida ao inseto $\left(\chi^{2}=47,52 ; G L=24 ; p=0,0029\right)$.

\section{DISCUSSÃO}

Teste "sem chance de escolha" com larvas. O resultado de dano nulo observado em 90,38\% das plantas testadas foi altamente significativo se comparado com trabalho similar. Ao testar larvas de $1^{\circ}$ ínstar de $P$. pertinax, Lucchini (1996) observou que em $62,12 \%$ de 66 plantas, as larvas não se alimentaram e com dois a três dias a mortalidade foi total. Em relação a sobrevivência das larvas de $1^{\circ}$ instar de $P$. confinis, Hoffmann \& Moscardi (1981) comentam que apenas uma sobreviveu até o $4^{\circ}$ dia em $B$. pilosa, mas não fazem referência sobre a alimentação.

Na categoria alimentação exploratória, o resultado de $5,77 \%$ foi menor do que o encontrado por Lucchini (1996) que obteve uma porcentagem de $18,18 \%$ das plantas testadas; e em ambos, houve mortalidade total das larvas no $1^{\circ}$ instar.

Em apenas uma planta $(1,92 \%)$ o dano foi classificado como fraco, este porcentagem tambémé menor do que a observada por Lucchini (1996) de 4,54\%; e em ambos os experimentos as larvas atingiram o $2^{\mathrm{O}}$ ínstar, mas nenhuma sobreviveu ao $3^{\mathrm{o}}$ instar.

Diferente desta pesquisa, na qual as larvas de $1^{0}$ instar não demonstraram interesse por Helianthus annuus L. e morreram até o $4^{\circ}$ dia de contato com a planta; Hoffmann \& MosCARDi (1981) comentam que uma das 30 larvas atingiu o $2^{\circ}$ ínstar ao se alimentar de Helianthus annuus L, mas os autores não fazem referencia ao tipo de dano causado à superfície foliar.

No presente experimento não foi obtido nenhum resultado que pudesse ser classificado como moderado. Entretanto Lucchini (1996) obteve 6,06\% de nível alimentar moderado (Tagetes erecta L., Cosmos sulphureus Cav., H. annuus e Brassica juncea L.) e algumas poucas larvas atingiram o estágio adulto; contudo afirmou que há baixa possibilidade de criação de $P$. pertinax nestas plantas.

Apenas em $S$. brasiliensis (1,92\%) o dano foi classificado como normal. Como esta foi a única planta com resultado de dano normal, pode-se afirmar que os resultados foram melhores que os obtidos por LuCCHINI (1996), que teve um dano normal em seis espécies vegetais (9,09\%), sendo que quatro delas pertenciam ao mesmo gênero da planta alvo B. pilosa, e as demais (Coreopsis sp. e C. sulphureus) pertencem à mesma tribo.

Nesta categoria ao avaliar-se a sobrevivência, das 60 larvas iniciais, 31,67\% atingiram a fase adulta; já HoFFMANn \& MosCARdi (1981) conseguiram que 56,66\% das larvas criadas no $S$. brasiliensis atingissem o estágio de adulto; e em Lucchini (1996), a viabilidade larva-adulto nas espécies de Bidens spp. foi maior que $85 \%$ e em Coreopsis sp. de $78 \%$. Supõe-se que esta taxa mais baixa de sobrevivência foi devido aos testes terem sido realizados em temperatura ambiente, diferente dos outros trabalhos que tiveram a temperatura controlada.

Teste "sem chance de escolha" com adultos. Ao aplicar o teste nos adultos observou-se semelhança na porcentagem de resultados nulos entre o presente trabalho e o de Lucchin (1996). Enquanto que o autor obteve resultado nulo em 79,10\% das plantas, o encontrado para este estudo foi de $82.60 \%$. Esta similaridade se estende a reação de desinteresse pelas plantas e alta taxa de mortalidade.

A alimentação exploratória representou 13,04\% do total das plantas testadas; em LuCCHINI (1996) a porcentagem foi menor (5,97\%); e HoFFmann \& Moscardi (1981) não obtiveram nenhum dano classificado como exploratório.

O dano classificado como fraco foi observado em $E$. sonchifolia, representando 2,17\% das 46 plantas; no trabalho de Lucchini (1996) o resultado foi similar, pois somente em duas plantas $(2,99 \%)$ 
Não foi obtido nenhum resultado que fosse classificado como moderado; mas ao se comparar com Lucchini (1996), $P$. pertinax apresentou alimentação moderada por $T$. erecta e C. sulphureus; com índice de sobrevivência de $25 \%$ em $T$. erecta e mortalidade total em C. sulphureus ao final do experimento.

Nos testes com $S$. brasiliensis, representando $2,17 \%$ das plantas testadas, os danos nas folhas foram classificados como normais devido a voracidade na alimentação, causando deterioração de grande parte da área foliar oferecida aos insetos. Assim como nos resultados dos testes com as larvas de $1^{\circ}$ instar, $P$. confinis mostrou-se mais especifico do que $P$. pertinax, pois este último encontrou nas espécies de Bidens spp. e Coreopsis sp. condições nutricionalmente adequadas para alimentação com alto índice de sobrevivência (Lucchini 1996).

No presente experimento a taxa de sobrevivência de adultos até o final dos 20 dias de teste foi de: $75 \% \mathrm{~S}$. brasiliensis, $65 \%$ E. sonchifolia, $45 \%$ B. oleracea var. capitata, $40 \%$ B. oleracea var. italica, $35 \%$ A. cepa, $10 \%$ B. pilosa, $5 \%$ B. pekinensis e $0 \% B$. oleracea var. acephala e $B$. oleracea var. botrytis.

Diferente dos resultados descritos por Lucchini (1996), onde $P$. pertinax realizou posturas em seis plantas diferentes no teste sem chance de escolha, $P$. confinis somente ovipositou nas folhas de $S$. brasiliensis, obtendo uma média de 127,20 ovos por placa-de-Petri, durante os 20 dias de observação. Mecanismos comportamentais permitem aos insetos herbívoros escolher o local onde depositar os ovos ou a progênie em plantas que oferecem melhores condições para o desenvolvimento da futura geração (Pizzamiglio-Gutierrez 2009). De um total de 615 ovos, $88,13 \%$ foram postos na face abaxial das folhas, $9,11 \%$ na face adaxial e 2,76\% postos diretamente nas placas-de-Petri. Havendo uma viabilidade de 73,01\%, ou seja, 449 ovos, deram origem a larvas de $1^{\circ}$ instar.

Bertels (1953) mencionou a presença de $P$. confinis sobre a batata-inglesa, mas os resultados desta pesquisa indicam que o inseto não demonstra interesse alimentar por estas espécies vegetais.

Teste de "múltipla escolha" com larvas. As nove plantas que obtiveram resultado diferente de nulo nos testes anteriores, participaram do teste de "múltipla escolha". Em 66,67\% não ocorreu nenhum tipo de dano. Foi observado que assim que as larvas de $1^{0}$ instar foram colocadas no centro da placa, dirigiramse imediatamente ao $S$. brasiliensis, ignorando as demais plantas presentes. Uma vez estabelecidas no $S$. brasiliensis, as larvas agregavam-se até o ínstar seguinte.

Em B. oleracea var. itálica e $A$. cepa, as larvas continuaram a apresentar algum tendência alimentar sendo os danos classificados como fraco $(11,11 \%)$ e exploratório $(11,11 \%)$, respectivamente. Apenas em S. brasiliensis o dano foi classificado como normal (11,11\%). Em trabalho anterior realizado com a espécie, os autores comentam que $P$. confinis mostrou-se especifico ao $S$. brasiliensis quando lhe foram ofertadas sete diferentes espécies vegetais, numa amostra de 13 (Hoffmann \& Moscardi 1980).

Insetos herbívoros são especializados em escolher plantas hospedeiras, que apresentem os requisitos fisiológicos e ecológicos adequados para completarem seu ciclo de vida (oviposição, alimentação e desenvolvimento larval, pupação, etc.) (WANG et al. 2008). Apesar de P. confinis demonstrar interesse alimentar por $E$. sonchifolia, B. oleracea (var. capitata e itálica) e A. cepa, provavelmente estas plantas não forneceram os nutrientes necessários para promover o desenvolvimento e a sobrevivência larval e nem as condições para oviposição pelas fêmeas.

Os resultados demonstram que alimentação normal, oviposição, sobrevivência e desenvolvimento de $P$. confinis estão restritos ao $S$. brasiliensis. E que além desta tendência a oligofagia, o inseto possui um conjunto de características adequadas para atuar no controle biológico, tais como: ser de fácil manuseio, tornando possível confinar um grande número de indivíduos em pequenos espaços; e possibilidade se obter várias gerações ao ano.

A colonização exagerada do $S$. brasiliensis em alguns ambientes indica que a pressão de seus inimigos naturais foi reduzida; portanto, $P$. confinis tem potencial para atuar como agente no controle biológico aplicado inundativo. Neste tipo de estratégia seleciona-se um inimigo natural endêmico e suficientemente específico para não constituir risco às outras plantas, visando um impacto negativo sobre a população da planta-alvo (PitelLi et al. 2003).

Tabela 1. Plantas selecionadas para os experimentos e resultados dos testes de especificidade hospedeira com larvas de $1^{\circ}$ ínstar e adultos de Phaedon confinis.

\begin{tabular}{|c|c|c|c|c|c|c|c|}
\hline & Espécie & Nome comum & $\begin{array}{c}\text { Sem } \\
\text { Chance } \\
\text { L1 }\end{array}$ & $\begin{array}{l}\text { Sobrevivência } \\
\text { (dias) / Estágio } \\
\text { desenvimento }\end{array}$ & $\begin{array}{l}\text { Sem } \\
\text { Chance } \\
\text { Adulto }\end{array}$ & $\begin{array}{l}\text { Sobrevivência } \\
\text { (até 2o dias) } \\
\text { (\%) }\end{array}$ & $\begin{array}{c}\text { Múltipla } \\
\text { Escolha } \\
\text { L1 }\end{array}$ \\
\hline \multicolumn{8}{|c|}{ ASTERACEAE } \\
\hline 1 & Aspilia setosa Griseb. & mal-me-quer & - & 4d/L1 & - & o & $\mathrm{N}$ \\
\hline 2 & Baccharis dracunculifolia DC. & vassourinha & - & $4 \mathrm{~d} / \mathrm{L} 1$ & - & o & $\mathrm{N}$ \\
\hline 3 & Bidens pilosa $\mathrm{L}$. & picão-preto & - & $2 \mathrm{~d} / \mathrm{L} 1$ & + & 10 & - \\
\hline 4 & Bidens subalternans DC. & picão-do-campo & - & $2 \mathrm{~d} / \mathrm{L} 1$ & - & o & $\mathrm{N}$ \\
\hline 5 & Calendula officinale $\mathrm{L}$. & calêndula & - & 4d/L1 & - & o & $\mathrm{N}$ \\
\hline 6 & Chicorium endivia $\mathrm{L}$. & escarola & - & $4 \mathrm{~d} / \mathrm{L} 1$ & - & o & $\mathrm{N}$ \\
\hline 7 & $\begin{array}{l}\text { Chrysanthemum leucanthemum } \\
\text { Linn }\end{array}$ & margarida & - & 4d/L1 & - & o & $\mathrm{N}$ \\
\hline 8 & Emilia sonchifolia (L.) DC. & bela-emília & + & 4d/L1 & ++ & 65 & - \\
\hline 9 & Eupatorium pauciflorum Kunth & cambará & - & $2 \mathrm{~d} / \mathrm{L} 1$ & - & o & $\mathrm{N}$ \\
\hline 10 & Galinsoga parviflora Cav. & picão-branco & - & 4d/L1 & - & o & $\mathrm{N}$ \\
\hline 11 & Hypochoeris radicata L. & almeirão-do-campo & - & 4d/L1 & - & 0 & $\mathrm{~N}$ \\
\hline 12 & Helianthus annuus L. & girassol & - & $4 \mathrm{~d} / \mathrm{L} 1$ & - & o & $\mathrm{N}$ \\
\hline \multirow{3}{*}{13} & \multirow{3}{*}{ Lactuca sativa $\mathrm{L}$. } & crespa verde & \multirow{3}{*}{-} & $6 \mathrm{~d} / \mathrm{L} 1$ & \multirow{3}{*}{ - } & o & \multirow{3}{*}{$\mathrm{N}$} \\
\hline & & crespa roxa & & 4d/L1 & & o & \\
\hline & & lisa verde & & $2 \mathrm{~d} / \mathrm{L} 1$ & & o & \\
\hline 14 & Mikania cordifolia (L. f.) Willd & guaco & - & $4 \mathrm{~d} / \mathrm{L} 1$ & - & o & $\mathrm{N}$ \\
\hline 15 & Senecio brasiliensis Less. & maria-mole & ++++ & 20d/Ad & ++++ & 75 & ++++ \\
\hline
\end{tabular}




\begin{tabular}{|c|c|c|c|c|c|c|c|}
\hline & Espécie & Nome comum & $\begin{array}{c}\text { Sem } \\
\text { Chance } \\
\text { L1 }\end{array}$ & $\begin{array}{l}\text { Sobrevivência } \\
\text { (dias) / Estágio } \\
\text { desenvimento }\end{array}$ & $\begin{array}{l}\text { Sem } \\
\text { Chance } \\
\text { Adulto }\end{array}$ & $\begin{array}{c}\text { Sobrevivência } \\
\text { (até 2o dias) } \\
\text { (\%) }\end{array}$ & $\begin{array}{c}\text { Múltipla } \\
\text { Escolha } \\
\text { L1 }\end{array}$ \\
\hline 16 & Sonchus oleraceus L. & chicória-brava & - & $2 \mathrm{~d} / \mathrm{L} 1$ & - & o & $\mathrm{N}$ \\
\hline 17 & $\begin{array}{l}\text { Tanacetum parthenium (L.) Sch. } \\
\text { Bip. }\end{array}$ & margaridinha & - & $4 \mathrm{~d} / \mathrm{L} 1$ & - & o & $\mathrm{N}$ \\
\hline 18 & Taraxacum officinalle Weber & dente-de-leão & - & $2 \mathrm{~d} / \mathrm{L} 1$ & - & o & $\mathrm{N}$ \\
\hline 19 & Vernonia sp. Screb. & vernonia & - & $2 \mathrm{~d} / \mathrm{L} 1$ & - & o & $\mathrm{N}$ \\
\hline \multicolumn{8}{|c|}{ ALLIACEAE } \\
\hline 20 & Allium cepa L. & cebola & + & $4 \mathrm{~d} / \mathrm{L} 1$ & + & 35 & + \\
\hline \multicolumn{8}{|c|}{ APIACEAE } \\
\hline 21 & Daucus carota $\mathrm{L}$. & cenoura & - & $2 \mathrm{~d} / \mathrm{L} 1$ & - & o & $\mathrm{N}$ \\
\hline 22 & Torilis sp. Jury S.L. & salsinha & - & 4d/L1 & - & o & $\mathrm{N}$ \\
\hline \multicolumn{8}{|c|}{ BRASSICACEAE } \\
\hline 23 & Brassica oleracea var. acephala L. & couve & + & 4d/L1 & - & o & - \\
\hline 24 & Brassica oleracea var. botrytis L. & couve-flor & - & $2 \mathrm{~d} / \mathrm{L} 1$ & + & o & - \\
\hline 25 & Brassica oleracea var. capitata $\mathrm{L}$. & repolho & - & $4 \mathrm{~d} / \mathrm{L} 1$ & + & 45 & - \\
\hline 26 & Brassica oleracea var. italica $\mathrm{L}$. & brócolis & ++ & $4 \mathrm{~d} / \mathrm{L} 2$ & + & 40 & ++ \\
\hline 27 & Brassica pekinensis Lour. & couve-chinesa & - & $6 \mathrm{~d} / \mathrm{L} 1$ & + & 5 & - \\
\hline 28 & Brassica rapa $\mathrm{L}$. & nabo branco & - & $2 \mathrm{~d} / \mathrm{L} 1$ & - & o & $\mathrm{N}$ \\
\hline 29 & Brassica juncea L. & mostarda & - & $2 \mathrm{~d} / \mathrm{L} 1$ & - & o & $\mathrm{N}$ \\
\hline \multicolumn{8}{|c|}{ CUCURBITACEAE } \\
\hline 30 & Sechium edule (Jacq.) Sw. & chuchu & - & 6d/L1 & - & o & $\mathrm{N}$ \\
\hline \multicolumn{8}{|c|}{ EUPHORBIACEAE } \\
\hline 31 & Manihot utilissima Pohl & mandioca & - & 4d/L1 & $\mathrm{N}$ & & $\mathrm{N}$ \\
\hline 32 & Ricinus comunnis $\mathrm{L}$. & mamona & - & 4d/L1 & - & o & $\mathrm{N}$ \\
\hline \multicolumn{8}{|c|}{ LEGUMINOSAE } \\
\hline 33 & Glycine $\max (\mathrm{L}$.) Merril & soja & - & 4d/L1 & - & o & $\mathrm{N}$ \\
\hline \multicolumn{8}{|c|}{ MORACEAE } \\
\hline 34 & Morus nigra $\mathrm{L}$. & amoreira & - & 4d/L1 & - & o & $\mathrm{N}$ \\
\hline \multicolumn{8}{|c|}{ MYRTACEAE } \\
\hline 35 & Eugenia uniflora L. & pitangueira & - & $2 \mathrm{~d} / \mathrm{L} 1$ & - & o & $\mathrm{N}$ \\
\hline 36 & Psidium guajava L. & goiabeira & - & $4 \mathrm{~d} / \mathrm{L} 1$ & $\mathrm{~N}$ & & $\mathrm{~N}$ \\
\hline 37 & Psidium cattleianum Sabine & araçazeiro & - & $2 \mathrm{~d} / \mathrm{L} 1$ & - & o & $\mathrm{N}$ \\
\hline \multicolumn{8}{|c|}{ POACEAE } \\
\hline 38 & Zea mays $\mathrm{L}$. & milho & - & 4d/L1 & - & o & $\mathrm{N}$ \\
\hline \multicolumn{8}{|c|}{ ROSACEAE } \\
\hline 39 & Fragaria sp. L. & morangueiro & - & $2 \mathrm{~d} / \mathrm{L} 1$ & - & o & $\mathrm{N}$ \\
\hline 40 & Prunus persica (L.) Batsh. & pessegueiro & - & $4 \mathrm{~d} / \mathrm{L} 1$ & - & o & $\mathrm{N}$ \\
\hline 41 & Rubus sellowii Cham. \& Schltdl. & amora-do-mato & - & $2 \mathrm{~d} / \mathrm{L} 1$ & - & o & $\mathrm{N}$ \\
\hline \multicolumn{8}{|c|}{ RUBIACEAE } \\
\hline 42 & Coffea arabica L. & café & - & 4d/L1 & - & o & $\mathrm{N}$ \\
\hline \multicolumn{8}{|c|}{ RUTACEAE } \\
\hline 43 & Citrus limom (L.) Burm. F. & limoeiro & - & $4 \mathrm{~d} / \mathrm{L} 1$ & $\mathrm{~N}$ & & $\mathrm{~N}$ \\
\hline 44 & Citrus reticulata Blanco & mexeriqueira & - & 2d/L1 & $\mathrm{N}$ & & $\mathrm{N}$ \\
\hline 45 & Citrus sinensis (L.) Osbeck & laranjeira & - & $2 \mathrm{~d} / \mathrm{L} 1$ & $\mathrm{~N}$ & & $\mathrm{~N}$ \\
\hline \multicolumn{8}{|c|}{ SAXIFRAGACEAE } \\
\hline 46 & Hydrangea macrophylla Ser. & hortênsia & - & 4d/L1 & - & o & $\mathrm{N}$ \\
\hline \multicolumn{8}{|c|}{ SOLANACEAE } \\
\hline 47 & Lycopersicon esculentum Mill. & tomateiro & - & $4 \mathrm{~d} / \mathrm{L} 1$ & $\mathrm{~N}$ & & $\mathrm{~N}$ \\
\hline 48 & Solanum tuberosum L. & batata-inglesa & - & $2 \mathrm{~d} / \mathrm{L} 1$ & - & o & $\mathrm{N}$ \\
\hline \multicolumn{8}{|c|}{ VERBENACEAE } \\
\hline 49 & Lantana camara L. & capitão-do-campo & - & $4 \mathrm{~d} / \mathrm{L} 1$ & - & o & $\mathrm{N}$ \\
\hline 50 & $\begin{array}{l}\text { Stachytarpheta cayennensis (Rich.) } \\
\text { Vahl }\end{array}$ & gervão & - & 2d/L1 & - & o & $\mathrm{N}$ \\
\hline
\end{tabular}




\section{REFERÊNCIAS}

Bertels, A., 1953. Pragas de solanáceas cultivadas. Agros 6: 154160.

Coombs, E., C. Mallory-Smith, L.C. Burrill; R.H. Callihan; R. Parker \& H. Radtke, 1997. Tansy Ragwort. Senecio jacobaea L. Pacific Northwest Extension Publication, 175: 1-8.

Cox, M.L., 1996. The unusual larva and adult of the Oriental Phaedon fulvescens Weise (Coleoptera: Chrysomelidae: Chrysomelinae): a potential biocontrol agent of Rubus in the Mascarenes. Journal of Natural History, 30:135-151.

Gazi, A.D.F.A, D. Gandolfo \& R.A. Pitelli, 2008. Preferência alimentar de adultos de Metriona elatior Klug (Coleoptera: Chrysomelidae, Cassidinae) por diferentes híbridos de Solanun melogena Linnaeus (Solanaceae). Acta Scientiarum - Biological Sciences, 30: 449-454.

Hoffmann, C.B. \& F. Moscardi, 1980. Controle biológico de Senecio brasiliensis Less com Phaedon confine (Klug, 1829) (Coleoptera: Chrysomelidae), p. 254-256. In: EMBRAPA. Centro Nacional de Pesquisa de Soja (Londrina, PR). Resultados de pesquisa de soja 1979/80.

Hoffmann, C.B. \& F. Moscardi, 1981. Teste de desenvolvimento de Phaedon confine (Klug, 1829) (Coleoptera: Chrysomelidae) em 24 hospedeiros, p.165-166. In: EMBRAPA. Centro Nacional de Pesquisa de Soja (Londrina, PR). Resultados de pesquisa de soja 1980/81.

Ilha, M.R.S., A.P. Loretti, S.S. Barros \& C.S.L. Barros, 2001. Intoxicação espontânea por Senecio brasiliensis (Asteraceae) em ovinos no Rio Grande do Sul. Pesquisa Veterinária Brasileira, 21: 123-138.

Lenteren, J.C. van., 2008. Biological control of weeds, p.51-6o. In: Lenteren, J.C. van. IOBC - Internet Book of Biological Control, version 5, 135p.

Lima, A. da Costa, 1954. Sobre espécies sul-americanas de Phaedon (Col. Chrysomelidae). Dusenia, 4: 428-432.

Lucchini, F., 1996. Especificidade hospedeira e aspectos biológicos de Phaedon pertinax Stal, 1860 (Coleoptera, Chrysomelidae), para o controle biológico de Bidens pilosa L. (Asteraceae). Tese de Doutorado (Área de Concentração Entomologia) Escola Superior de Agricultura "Luiz de Queiroz”, Piracicaba, USP/ESALQ, $75 \mathrm{p}$.

McEvoy, P., C. Cox \& E. Coombs, 1991. Successful biological control of ragwort, Senecio jacobaea, by introduced insects in Oregon. Ecological Applications, 1: 430-422.

McFadyen, R.E.C, 1998. Biological control of weeds. Annual Review of Entomology, 43: 369-393.

\section{Como citar este artigo:}

Milléo, J., J.P. Castro \& J.H. Pedrosa-Macedo, 2011. Teste de especificidade hospedeira de Phaedon confinis (Coleoptera, Chrysomelidae), um potencial agente de biocontrole de Senecio brasiliensis (Asteraceae). EntomoBrasilis, 4(2): 61-66. www.periodico.ebras.bio.br/ojs
Mendes, M.M., M.L. Leite, G.H. Corrêa \& J. Milléo, 2005. Entomofauna associada ao Senecio brasiliensis Less (Asteraceae), e Phaedon confinis (Insecta; Coleoptera; Chrysomelidae) como possível agente controlador desta planta tóxica. Publicatio UEPG - Ciências Exatas e da Terra, Ciências Agrárias e Engenharias, 11: 45-53.

Milléo, J., G.H. Corrêa, M.L. Leite \& J.H. Pedrosa-Macedo, 2006. Comportamento e ciclo de vida de Phaedon confinis (Coleoptera, Chrysomelidae) em condições de laboratório. Revista Brasileira de Entomologia, 50: 419-422.

Pitelli, R.A., G.F. Nachtigal \& R.L.C.M. Pitelli, 2003. Controle biológico de plantas daninhas. In: Manzanillo: Congreso Latinoamericano de Malezas, 16: 518-524.

Pizzamiglio-Gutierrez, M.A. 2009. Interações Inseto-Planta, p. 211-249. In: Panizzi, A.R. \& J.R.P. Parra. Bioecologia e nutrição de insetos: Base para o manejo integrado de pragas. Brasília, DF: Embrapa Informações Técnicas, 1161p.

Riet-Correa, F., M.C. Méndez \& A.L. Schild, 1993. Intoxicações por Plantas e Micotoxicoses em Animais Domésticos. Montevidéu, Editorial Hemisfério Sur, 340p.

Rissi, D.R., R.R. Rech, F. Pierezan, A.L. Gabriel, M.E. Trost, J.S. Brum, G.D. Kommers \& C.S.L. Barros, 2007. Intoxicações por plantas e micotoxinas associadas a plantas em bovinos no Rio Grande do Sul: 461 casos. Pesquisa Veterinária Brasileira, 27: 261-268.

Silva, A.G.A., C.R. Gonçalves, D.M. Galvão, A.J.L. Gonçalves, J. Gomes, M.N. Silva \& L. Simoni, 1968. Quarto catálogo dos insetos que vivem nas plantas do Brasil: seus parasitos e predadores. Rio de Janeiro, Ministério da Agricultura, Depto. de Defesa e InspeçãoAgropecuária, pt. 2, t.1, 621p.

Wang, Y., J. Ding \& G. Zhang, 2008. Gallerucida bifasciata (Coleoptera: Chrysomelidae), a potential biological control agent for Japonese knotweed (Fallopia japonica). Biocontrol Science and Technology, 18:59-74.

Wapshere, A.J., 1974. A strategy for evaluating the safety of organisms for biological weed control. Annals of Applied Biology 77: 20-211.

Zhang, Y., J.L. Hanula \& J. Sun, 2008. Host specificity of Argopistes tsekooni (Coleoptera: Chrysomelidae), a potential biological control agent of Chinese Privet. Journal of Economic Entomology, 101: 1146-1151.

Recebido em: 26/10/2010

Aceito em: 14/12/2010

$* * * * * * * * * * * *$
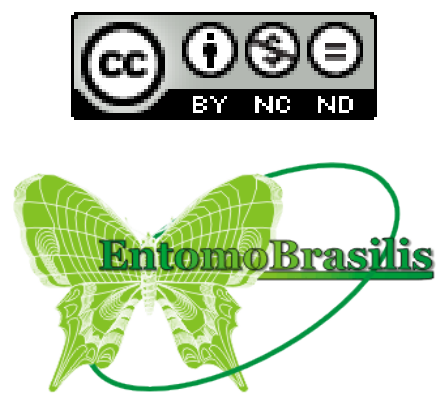